\title{
A metabolomics approach to uncover the effects of grain diets on rumen health in dairy cows
}

\author{
F. Saleem, ${ }^{\star}$ B. N. Ametaj,,${ }^{1}$ S. Bouatra, ${ }^{\star}$ R. Mandal, ${ }^{\star}$ Q. Zebeli, $\neq$ S. M. Dunn, $\dagger$ and D. S. Wishart ${ }^{\star 1}$ \\ *Departments of Biological and Computing Sciences, and \\ †Department of Agricultural, Food and Nutritional Science, University of Alberta, Edmonton, AB, Canada TG6 2P5 \\ łInstitute of Animal Nutrition, Department for Farm Animals and Veterinary Public Health, Vetmeduni, Veterinaerplatz 1, 1210 Vienna, Austria
}

\begin{abstract}
Dairy cows fed high-grain diets during early lactation have a high incidence of metabolic disorders. However, the precise mechanism(s) of how grain feeding causes disease is not clear. In an effort to understand how this diet transition alters the rumen environment and potentially leads to certain metabolic disorders in dairy cattle, we undertook a comprehensive, quantitative metabolomic analysis of rumen fluid samples from dairy cows fed 4 different diets. Using a combination of proton nuclear magnetic resonance spectroscopy, gas chromatography-mass spectrometry, and direct flow injection tandem mass spectroscopy, we identified and quantified 93 metabolites in rumen samples taken from 8 dairy cows fed graded amounts of barley grain (i.e., $0,15,30$, and $45 \%$ of diet dry matter). We also studied temporal changes in the rumen by studying metabolite concentration differences between the first day and the last day of each diet phase following the diet adaptation period. Multivariate analysis showed that rumen metabolites arising from the diet containing $45 \%$ barley grain were clearly different from those containing 0 , 15 , and $30 \%$ barley grain. Likewise, a clear separation of the metabolic composition of the ruminal fluid was evident at the beginning and at the end of each diet phase - contrary to the belief that $11 \mathrm{~d}$ are suitable for the adaptation of cows to high-grain diets. High-grain diets $(>30 \%)$ resulted in increased rumen fluid concentrations of several toxic, inflammatory, and unnatural compounds including putrescine, methylamines, ethanolamine, and short-chain fatty acids. Perturbations in several amino acids (phenylalanine, ornithine, lysine, leucine, arginine, valine, and phenylacetylglycine) were also evident. The present study confirms and greatly extends earlier observations on dietary effects on rumen fluid composition and shows that the use of multiple
\end{abstract}

\footnotetext{
Received February 1, 2012.

Accepted July 8, 2012.

${ }^{1}$ Corresponding authors: burim.ametaj@ualberta.ca and david. wishart@ualberta.ca
}

metabolomic platforms permits a far more detailed understanding of metabolic causes and effects. These results may improve our understanding of diet-related rumen metabolism and the influence of grain on the overall health of dairy cattle.

Key words: dairy cow, metabolomics, rumen fluid, barley grain

\section{INTRODUCTION}

Early lactation is a very critical phase in the nutrition and management of dairy cows. During early lactation dairy cows are fed diets with a high proportion of cereal grains to meet the high energy and nutrient demands for milk production and alleviate any negative energy balance. Because cereal grains are rich in starch, and because starch is rapidly degraded in the rumen it releases large amounts of VFA, including acetate, propionate, and butyrate, as well as other organic acids such as lactate (Iqbal et al., 2009). The rapid release of large amounts of these short-chain FA (SCFA) causes rumen metabolic disorders such as SARA, which is associated with major changes in the rumen environment (Zebeli et al., 2008). Multiple lines of evidence support the idea that feeding large amounts of grain contributes to the high incidence of diseases in dairy cattle (reviewed by Ametaj et al., 2010a). Recent reports have demonstrated that feeding high proportions of grain causes major changes in the composition of rumen microbiota in favor of gram-negative bacteria (i.e., Escherichia coli and Megasphaera elsdenii; Khafipour et al., 2009; Fernando et al., 2010) and the composition of rumen fluid metabolites (Ametaj et al., 2010b).

Despite much progress made in the understanding and prevention of SARA in dairy cattle (Stone, 2004), the etiopathogenesis of SARA and its consequences to the host health still remain unknown. Recent advances made using throughput, quantitative metabolomic methods (Psychogios et al., 2011) have opened new avenues to elucidate the associations of feeding starchrich diets and rumen health. Preliminary data from our team suggests that in addition to endotoxin, several 
other metabolites released into the ruminal fluid due to high-grain diets might also contribute to disease development or disease exacerbation.

In particular, a pilot nuclear magnetic resonance (NMR)-based metabolomic study conducted by our group in 2009 looked at the rumen fluid from dairy cows fed graded amounts of cereal grain (Ametaj et al., 2010b). Results from this study showed significant elevations of several harmful or potentially harmful compounds in the rumen fluid including methylamine, nitrosodimethylamine, and ethanol with increasing amounts of dietary grain. We also pointed out how these compounds might play a role in the development of some periparturient diseases in dairy cows (Ametaj et al., 2010b). In an effort to confirm and extend these studies, we have undertaken a much more comprehensive, quantitative metabolomic analysis of this same dairy cow cohort, applying much improved metabolite detection techniques, including proton NMR $\left({ }^{\mathbf{1}} \mathbf{H}\right.$ NMR) spectroscopy, GC-MS, and direct flow injection (DFI) tandem mass spectrometry (MS/MS). We have also performed significantly more assays on a larger number of samples covering many more time points to ascertain the temporal effects of diet adaptation (washin) and washout. By using a variety of sophisticated multivariate and metabolic pathway analyses we have been able to confirm several our pilot study results, but have also identified several additional rumen fluid compounds and rumen metabolic perturbations that might be involved in periparturient disease etiology. This study also provides a more comprehensive view of the diet-induced symbiotic effects between dairy cows and their microbiota that may help unravel the effects of diet on periparturient diseases (Ametaj et al., 2010b). We also show that the use of these modern metabolomic techniques could also prove helpful in the development and validation of new disease biomarkers for a variety of dairy cattle diseases.

\section{MATERIALS AND METHODS}

\section{Animals and Diets}

Eight ruminally cannulated (100-mm diameter; Bar Diamond Inc., Parma, ID) primiparous and clinically healthy Holstein cows were used in a replicated $4 \times$ 4 Latin square design at the University of Alberta's Dairy Research and Technology Centre (Edmonton, $\mathrm{AB}$, Canada). The total experimental period was 21 $\mathrm{d}$, with the first $11 \mathrm{~d}$ serving as a diet adaptation period. Based on similar feeding experiments, an adaptation period of $11 \mathrm{~d}$ was considered sufficient to allow wash-in of the experimental diets before metabolomic measurements. The remaining $10 \mathrm{~d}$ were allocated for collection of rumen fluid samples. At the start of the experiment, the cows were at (mean \pm SD) $60 \pm 15 \mathrm{~d}$ postpartum. All cows were fed the same basic ration, which was supplemented with increasing proportions of barley grain at $0,15,30$, or $45 \%$ in DM. The cows were housed in tiestalls with free access to water and were fed once daily at $0800 \mathrm{~h}$ and milked twice at 0500 and 1600 h, with milk yield recorded electronically. Daily rations were fed as TMR to meet or exceed the requirements of a 680-kg lactating cow as per NRC (2001) guidelines.

All experimental procedures were approved by the University of Alberta Animal Policy and Welfare Committee, and animals were cared for in accordance with the guidelines of the Canadian Council on Animal Care (CCAC, 1993). Data related to the endotoxin concentrations and $\mathrm{pH}$ of the rumen fluid, plasma acute-phase proteins, and milk production for this cohort of animals have been reported previously (Emmanuel et al., 2008; Ametaj et al., 2010b).

Estimated protein and energy contents were designed to be similar across the different diets. Ingredients and nutrient composition of TMR containing increasing proportions of rolled barley grain are given in Emmanuel et al. (2008). Individual feed intake was recorded daily throughout the 10-d rumen collection period by determining the difference between the total daily feed given to each cow with the feed refusals the next morning. The daily feed intake of cows is also reported previously (Emmanuel et al., 2008). All cows remained healthy during the entire experimental period and their health was monitored daily based on their daily feeding (i.e., DMI) and milking (i.e., milk yield) behavior as well as for clinical signs of disease by a veterinary technician.

\section{Rumen Fluid Collection}

For this study, a set of total 64 rumen fluid samples were collected from 8 cannulated cows for both d 1 and 10 of the measurement period (i.e., d 12 and 21), yielding a total of $\mathrm{n}=16$ for each of the 4 dietary treatments. All rumen fluid samples $(100 \mathrm{~mL}$ each) were collected through a cannula using a tube fitted with a strainer and a syringe, which were connected to a 140$\mathrm{mL}$ sterile plastic container. Rumen fluid samples were collected 15 to 30 min before the morning feeding from all cows. The $\mathrm{pH}$ of the rumen fluid was determined immediately after collection by a mobile $\mathrm{pH}$ meter (Accumet AP61; Fischer Scientific, Ottawa, ON, Canada). Subsequently, rumen fluid samples were centrifuged at $6,000 \times g$ for 15 min (Rotanta $460 \mathrm{R}$; Hettich Zentrifugan, Tuttlingen, Germany), and the supernatant was filtered through a disposable $0.22-\mu \mathrm{m}$ sterile, pyrogenfree filter (Fischer Scientific, Fairlawn, NJ). All rumen 
fluid samples were frozen at $-20^{\circ} \mathrm{C}$ within $2 \mathrm{~h}$ to minimize any possible metabolite degradation. All rumen fluid samples were thawed on ice for approximately $2 \mathrm{~h}$ before metabolomic analysis.

\section{Endotoxin Analysis of Rumen Fluid}

The concentration of cell-free endotoxin was determined by the Pyrochrome Limulus amebocyte lysate assay according to manufacturer instructions (Associates of Cape Cod Inc., East Falmouth, MA) as described previously (Emmanuel et al., 2008). Samples were tested in duplicate, and the optical density values read on a microplate spectrophotometer (Spectramax 190; Molecular Devices Corp., Sunnyvale, CA) at a wavelength of $405 \mathrm{~nm}$. Intraassay coefficient of variation was $<10 \%$ for all assays.

\section{NMR Compound Identification and Quantification}

All ${ }^{1} \mathrm{H}$ NMR spectra of rumen samples were collected on a either a $500 \mathrm{MHz}$ (Varian Inc., Palo Alto, CA) spectrometer using the first transient of the tnnoesypresaturation pulse sequence. The resulting ${ }^{1} \mathrm{H}$ NMR spectra were processed and analyzed using the Chenomx NMR Suite Professional software package version 6.0 (Chenomx Inc., Edmonton, AB, Canada), as previously described (Ametaj et al., 2010b). Further details on the NMR sample preparation and NMR data acquisition are provided in the supplemental information (available online at http://www.journalofdairyscience.org/).

\section{GC-MS Compound Identification and Quantification}

Prior to analysis by GC-MS, rumen fluid samples were extracted to separate polar metabolites from nonpolar (i.e., lipophilic) metabolites. The rumen extraction and derivatization protocol for the GC-MS work was adapted from previously described methods (Jiye et al., 2005). For polar metabolites, an aliquot of $100 \mu \mathrm{L}$ of ruminal fluid containing $2 \mu \mathrm{L}$ of ribitol in water $(20$ $\mu \mathrm{g} / \mathrm{mL}$ ), as an internal standard, was extracted with $800 \mu \mathrm{L}$ of cold HPLC-grade methanol:double-distilled water $(8: 1 \mathrm{vol} / \mathrm{vol})$ and vortexed for $1 \mathrm{~min}$. The sample was kept at $4^{\circ} \mathrm{C}$ for 20 min and then centrifuged at $6,000 \times g$ for $8 \mathrm{~min}$. After centrifugation, $200 \mu \mathrm{L}$ of the supernatant were evaporated to dryness using a SpeedVac concentrator (SDC-100-H; Savant Instruments Inc., Farmingdale, NY). After evaporation, all samples were derivatized with $\mathrm{N}$-methyl-N-trifluoroacetamide (MSTFA) with $1 \%$ trimethylchlorosilane (TMCS) and the resulting extracts were separated and analyzed using an Agilent 5890 Series II GC-MS unit (Agilent Technologies Inc., Palo Alto, CA) operating in elec- tron impact ionization mode. Further details on the extraction, derivatization, separation and GC-MS data analysis are provided in the Supplemental Information (available online at http://www.journalofdairyscience. $\operatorname{org} /)$.

\section{Direct Flow Injection MS/MS Compound Identification and Quantification}

To determine concentrations of amino acids, sugars, acylcarnitines, sphingolipids, and glycerophospholipids in the rumen samples, the Absolute $I D Q$ kit p150 (Biocrates Life Sciences AG, Innsbruck, Austria) was prepared as described in the manufacturer's protocol. The method involves derivatization and extraction of analytes from the rumen samples, along with selective mass-spectrometric detection and quantification via multiple reaction monitoring. Isotope-labeled internal standards are integrated into the kit plate filter to facilitate metabolite quantification (see Supplemental Information for additional information, available online at http://www.journalofdairyscience.org/). The kit was originally validated for plasma/serum samples but cross-validation with other techniques $\left({ }^{1} \mathrm{H}\right.$ NMR and GC-MS) also showed excellent concordance with the kit's results with rumen fluid.

\section{Statistical Analyses}

Metabolite concentrations determined by endotoxin assays, ${ }^{1} \mathrm{H}$ NMR, GC-MS, and DFI-MS/MS experiments were compiled and cross-checked (to ensure that compound concentrations measured by 2 or more analytical platforms were consistent). For all statistical analyses, we only included those metabolites that were quantified in every rumen sample. Parametric analysis of the data was conducted using the MIXED procedure of SAS (SAS Institute Inc., Cary, NC; version 9.1.3) according to the following model:

$$
\begin{gathered}
Y_{i j k l m n}=\mu+p_{i}+a_{j}+a(s)_{j k}+d_{l} \\
+t_{m}+(d t)_{l m}+e_{i j k l m n},
\end{gathered}
$$

where $Y_{i j k l m n}$ is the observation for dependent variables, $\mu$ represents the population mean, $p_{i}$ is the fixed effect of period $i$ ( $i=1$ to 4$), a_{j}$ is the fixed effect of cow $j(j$ $=1$ to 8$), a(s)_{j k}$ is the random effect of cow $j$ within the square $k(k=1$ to 2$), d_{l}$ is the fixed effect of measurement day $l(l=1$ to 2$), t_{m}$ represents the fixed effect of diet $m(m=1$ to 4$),(d t)_{l m}$ is the diet by day interaction, and $e_{i j k l m n}$ is the residual error assumed to be normally distributed. The covariance structure of the repeated measures at different days for response vari- 
ables was modeled as first-order autoregressive, which provided the smallest values of the fit statistics based on the Bayesian information criteria. To test linear or quadratic effects of treatment on blood variables, the orthogonal contrasts with the CONTRAST statement of SAS (SAS Institute Inc.) were used. Data are shown as least squares means with respective standard errors of the means. Significance was declared at $P \leq 0.05$; a tendency was considered at $0.05<P \leq 0.10$.

Integrated concentration tables for 64 samples [n $=8$ for each day (d 1 and 10), yielding a total of $n$ $=16$ for each of the 4 dietary treatments] were then imported into the MetaboAnalyst web server (http:// www.metaboanalyst.ca; Xia et al., 2009) for multivariate analysis. Data integrity checks and data normalization were performed against the baseline metabolite concentrations measured for the $0 \%$-grain diet. This was done by first creating a dummy baseline sample by averaging all 16 samples collected at $0 \%$. After this normalization step, all samples were normalized against this reference sample using probabilistic quotient normalization procedures (Dieterle et al., 2006; Xia et al., 2009). The compound normalization was conducted using autoscaling, which made all compound variances equal to 1 . After this normalization step, the data were analyzed by multivariate statistical analysis using principal components analysis (PCA). Principal components analysis is an unsupervised clustering technique that can be used to examine the intrinsic variation in a data set and to reduce the dimensionality or complexity of the data. Scores plots were used to show the similarities and differences among the data sets. In a scores plot, observations (i.e., rumen samples) exhibiting metabolic similarities are clustered closer together, whereas those that are metabolically or chemically different are placed further apart. Loading plots were also used to reveal which variables (i.e., rumen fluid metabolites) were most responsible for the variation within the data set. Apart from revealing any similarities or separation trends among the data, PCA also removes any unwanted or systematic information (noise) from the data set. The axes of the scores and loading plots represent unique linear independent combinations of the observations and the variables, called principal components (PC), which are orthogonal to each other. Usually, the first $2 \mathrm{PC}$ (PC 1 and PC 2) capture most of the intrinsic variation in the data. In addition to this PCA study, another multivariate technique known as partial least squares discriminant analysis (PLS-DA) was performed using MetaboAnalyst (Xia et al., 2009). Partial least squares discriminant analysis is a supervised (as opposed to a nonsupervised) pattern recognition method that aims at maximizing any discriminating variation between predefined classes. The PLS-DA results were visualized using the first $2 \mathrm{PC}$ of the scores plot to identify characteristic trends or grouping among cows on the different diets. The corresponding loading plot was used to determine the metabolites most responsible for separation in the PLS-DA scores plot. Based on the PLS-DA results, metabolites were plotted according to their importance in separating the dietary groups and each metabolite received a value called variable importance in the projection (VIP). Variable importance in the projection values $>1$ suggest that the metabolite is significantly involved in the separation of groups (Szeto et al., 2010).

Hierarchical clustering analysis (HCA) with Euclidean distance measures and an average linkage method was also performed to explore the presence of clustering patterns among the rumen fluid metabolites. This was also done via MetaboAnalyst. The expression patterns and a heat map of each variable were categorized using an average linkage hierarchical clustering program (Seo, 2005).

\section{RESULTS}

\section{${ }^{1} \mathrm{H}$ NMR Compound Identification and Quantification}

Figure S1 (in the Supplemental Information, available online at http://www.journalofdairyscience.org/) shows the characteristic ${ }^{1} \mathrm{H}$ NMR spectra of ruminal fluid corresponding to dairy cows on diets with 0,15 , 30 , and $45 \%$ barley grain. All NMR spectra were collected on ruminal fluid samples obtained on d 12 and 21 of the experiment (d 1 and 10 of the measurement period). The NMR spectrum of rumen is remarkably simple and surprisingly uncomplicated, which made the identification and quantification of rumen metabolites relatively easy. Typically $98 \%$ of all visible peaks were assigned to a compound and more than $95 \%$ of the spectral area could be routinely fitted using the Chenomx spectral analysis software (Ametaj et al., 2010b). Most of the visible peaks can be annotated with a compound name. A total of 64 rumen sample (16 samples from each diet i.e., $0,15,30$, and $45 \%$ barley grain) were analyzed. An average of 48 compounds were unambiguously identified and quantified in each sample. Feeding dairy cows with increasing proportions of barley grain was associated with concentration increases of several rumen metabolites, including ethanol, ethanolamine, 3-hydroxybutyrate, methylamine (MA), dimethylamine, N-nitrosodimethylamine, glucose, propionate, butyrate, alanine, maltose, uracil, xanthine, phenylacetylglycine, and phenylacetate $(P<0.05)$. On the other hand, greater proportions of barley grain in the diet resulted in lower concentrations of 1,3-dihydroxyacetone and 3-phenylpropionate (3-PP) in the rumen 
fluid (Table 1). The complete list of average compound concentrations with their respective standard errors of the means is shown in Table 1.

\section{GC-MS Compound Identification and Quantification}

Typical high-resolution GC-MS total ion chromatograms of the ruminal fluid corresponding to diets with $0,15,30$, and $45 \%$ barley grain are shown in Figure S2 (in the Supplemental Information, available online at http://www.journalofdairyscience.org/). As can be seen in this figure, many of the visible peaks can be annotated with a compound name; however, approximately $40 \%$ of these peaks remain unidentified. This relatively low level of coverage is a common problem in global or untargeted GC-MS metabolomics studies. Whereas some of these peaks may be due to derivatization byproducts or degraded metabolites, the lack of a comprehensive GC-MS library for rumen fluid metabolites [the National Institute of Standards and Technology (NIST) mass spectra library contains only a small portion of metabolically relevant compounds; http://www.nist. gov/srd/nist1a.cfm] also limits the attainable coverage from automated library search algorithms. The use of other commercially available reference libraries for GC-MS (i.e., the Fiehn GC-MS library from Agilent, http://www.agilent.com/chem/fiehnlibrary) might have provided a slightly better and more complete coverage of the rumen metabolites. In total, 27 metabolites were quantified via GC-MS of which 10 were unique to this particular platform (Table 2). Comparisons between the NMR and GC-MS measured concentrations (across the 20 compounds that were quantified by both techniques) showed generally good agreement (within 20 to $30 \%$ of each other; data not shown). The ANOVA tests showed 8-fold and 2-fold increases in the rumen concentrations of putrescine and urea $(P<0.001)$, respectively, when feeding cows a $45 \%$ barley grain diet (Table 2). Also, increasing the amount of grain in the diet increased the concentrations of 4-aminobutyrate and several amino acids in the rumen fluid, including methionine, phenylalanine, and threonine. In contrast, the concentration of hydrocinnamic acid decreased in cows fed larger amounts of grain (Table 2).

\section{DFI-MS/MS Compound Identification and Quantification}

The DFI-MS /MS targeted analysis using the Biocrates Absolute $I D Q$ kit provided quantitative results for 45 metabolites (6 acylcarnitines, 12 amino acids, hexose, 15 phospatidylcholines, 9 sphingolipids, and 1 lysophosphatidylcholine; Table 3). From the 15 measured phospatidylcholines, only 4 provided quantitative data in all samples, whereas the remaining 11 were below the limit of detection in some rumen samples. Overall, results of ANOVA indicated that increased proportions of barley grain in the diets of dairy cows were associated with decreases in the rumen concentrations of several phosphatidylcholines and an elevated concentration of rumen fluid hexoses.

\section{Diet-Dependent Multivariate Analysis of Rumen Metabolites}

Our data show that feeding dairy cows increasing proportions of barley grain was associated with changes in the concentrations of several metabolites. To visualize the difference among the metabolites data, we carried out PCA. A PCA analysis of the complete set of 93 metabolites that were quantified in 64 rumen samples showed that the first 2 PC cover $32 \%$ of the observed variance in the sample set (Figure 1a). The PCA scores plot revealed the differences corresponding to cows fed different amounts of barley grain, which appeared to be well separated in both PC 1 and PC 2. The clusters corresponding to rumen metabolite profiles from diets with 0 and $15 \%$ grain strongly overlapped with each other. However, the clusters representing the $45 \%$ grain diets were spaced further apart from those corresponding to the 0,15 , and $30 \%$ grain diets. However, the cluster of cows fed $30 \%$ grain is located relatively far from the $0 \%$ cluster but very close to the $15 \%$ cluster (Figure 1a).

To visualize the individual rumen fluid metabolites responsible for the variation of the first $\mathrm{PC}$ (PC 1 and PC 2), we used the corresponding loading plot (Figure 1b). This scatter plot gives a graphical representation of the extent to which each metabolite accounts for the variance in the data and shows the relationships/ correlations between the different rumen metabolites. Metabolites with the same distance from 0 and with similar directions are positively correlated, whereas those in the opposite direction are negatively correlated. For example, MA, putrescine, hypoxanthine, glucose, and the bacterial membrane component LPS (or endotoxin) are positively correlated to each other with the $45 \%$ diet. These metabolites, on the other hand, are negatively correlated with $\mathrm{pH}$ and acetate. Those rumen metabolites that are positively associated with each other in the $45 \%$ diet appear to play an important role in the separation along the PC 1 axis. The metabolic consequences of the various diets (i.e., $0,15,30$, and $45 \%$ barley grain) were more clearly visible using PLS-DA. The PLS-DA scores plot revealed that it was possible to discriminate not only the 30 and $45 \%$ barley grain diets form each other, but more interestingly to separate the 0 and $15 \%$ diets (Figure 
Table 1. Concentration ( $\mu M$, unless otherwise stated) of rumen metabolites in dairy cows fed graded amounts of barley grain determined by nuclear magnetic resonance (LSM $\pm \mathrm{SEM} ; \mathrm{n}=8$ )

\begin{tabular}{|c|c|c|c|c|c|c|c|c|}
\hline Metabolite $^{1}$ & $\begin{array}{l}\text { Metabolic } \\
\text { pathway }^{2}\end{array}$ & \multicolumn{4}{|c|}{ Barley grain proportion ${ }^{3}$ (\% of diet DM) } & SEM & \multicolumn{2}{|c|}{$P$-value ${ }^{4}$} \\
\hline \multicolumn{9}{|l|}{ AA metabolism } \\
\hline 3-Phenylpropionate & FM & 626.8 & 523.6 & 482.5 & 410.6 & 25.7 & $<0.01$ & 0.54 \\
\hline Alanine & FM, PPM & 212.1 & 234.9 & 323.4 & 480.1 & 12.1 & $<0.01$ & $<0.01$ \\
\hline Aspartate & TCA, BM, SMB & 126 & 132.7 & 196.4 & 179.6 & 12.3 & $<0.01$ & 0.34 \\
\hline Glycine & PPM, SMB, GM & 119.8 & 130 & 177.6 & 189.8 & 12.5 & $<0.01$ & 0.93 \\
\hline Histidine & PPP, SMB & 48 & 42.9 & 55.1 & 28.8 & 3.46 & $<0.01$ & $<0.01$ \\
\hline Isobutyrate $(\mathrm{m} M)$ & PPM, PDA & 1.42 & 1.45 & 1.67 & 1.81 & 0.08 & $<0.01$ & 0.46 \\
\hline Isoleucine & SMB, TCA & 173.8 & 151.8 & 266.9 & 182.9 & 13 & 0.01 & 0.02 \\
\hline Isovalerate $(\mathrm{m} M)$ & $\mathrm{PDA}$ & 1.09 & 0.9 & 1.08 & 1.13 & 0.07 & 0.36 & 0.11 \\
\hline Leucine & SMB, TCA & 125.8 & 119.2 & 182.8 & 191.3 & 12.4 & $<0.01$ & 0.51 \\
\hline Lysine & TCA, PM & 117.9 & 118.9 & 163.8 & 231.8 & 8.28 & $<0.01$ & $<0.01$ \\
\hline \multicolumn{9}{|l|}{ Butanoate metabolism } \\
\hline 3-Hydroxybutyrate & $\mathrm{BM}$ & 63.3 & 78.6 & 89.4 & 109.9 & 5.7 & $<0.01$ & 0.65 \\
\hline 4-Hydroxybutyrate & $\mathrm{BM}$ & 206.2 & 216.9 & 253.1 & 239.4 & 17.5 & 0.08 & 0.48 \\
\hline Acetoacetate & $\mathrm{BM}, \mathrm{TM}, \mathrm{PM}$ & 67.7 & 64.1 & 77.9 & 80.1 & 4.33 & 0.01 & 0.5 \\
\hline Butyrate $(\mathrm{m} M)$ & $\mathrm{BM}$ & 9.38 & 8.1 & 8.03 & 15.13 & 0.45 & $<0.01$ & $<0.01$ \\
\hline Fumarate & $\mathrm{BM}, \mathrm{BOP}, \mathrm{TCA}$ & 8.8 & 11.2 & 7.5 & 11.7 & 0.72 & 0.12 & 0.24 \\
\hline \multicolumn{9}{|l|}{ Caffeine metabolism } \\
\hline Caffeine & $\mathrm{CM}$ & 22.8 & 16.2 & 25.5 & 16.6 & 1.58 & 0.19 & 0.47 \\
\hline \multicolumn{9}{|l|}{ Glycolysis/gluconeogenesis } \\
\hline 1,3-Dihydroxyacetone & GLM, GL & 16.6 & 6.5 & 6.8 & 6.3 & 0.68 & $<0.01$ & $<0.01$ \\
\hline Acetate $(\mathrm{m} M)$ & $\mathrm{AM}, \mathrm{GL}$ & 60.1 & 56.3 & 54.6 & 64.27 & 1.47 & 0.06 & $<0.01$ \\
\hline Ethanol & $\mathrm{AM}, \mathrm{GL}$ & 202.4 & 241.1 & 355.2 & 425.1 & 42.4 & $<0.01$ & 0.76 \\
\hline Glucose $(\mathrm{m} M)$ & GL, GNG & 0.52 & 0.75 & 1.1 & 2.43 & 0.11 & $<0.01$ & 0.01 \\
\hline Glycerol & GaM, GNG & 144 & 157.7 & 236 & 265.5 & 12.8 & $<0.01$ & 0.54 \\
\hline Lactate & $\mathrm{PM}, \mathrm{CC}$ & 156.9 & 173.5 & 282 & 180.4 & 17.2 & 0.02 & $<0.01$ \\
\hline \multicolumn{9}{|l|}{ Nucleotide metabolism } \\
\hline Hypoxanthine & PPM & 52.3 & 76.1 & 85.1 & 205 & 11.5 & $<0.01$ & $<0.01$ \\
\hline Ribose & PPM, AAM & 311.4 & 322.9 & 406.5 & 254.5 & 17.8 & 0.28 & $<0.01$ \\
\hline Uracil & PPM, CF, MM & 113.2 & 142.5 & 234.6 & 270.2 & 16.7 & $<0.01$ & 0.85 \\
\hline Xanthine & PPM, CF, SMB & 59.9 & 91.7 & 116.6 & 177.6 & 10.6 & $<0.01$ & 0.18 \\
\hline \multicolumn{9}{|l|}{ TCA cycle } \\
\hline Fumarate & TCA, AAM, PPS & 8.8 & 11.2 & 7.5 & 11.7 & 0.72 & 0.12 & 0.24 \\
\hline Nicotinate & TCA, SMB, AAM & 37.4 & 43 & 62.1 & 41.1 & 3.07 & 0.03 & $<0.01$ \\
\hline Propionate $(\mathrm{m} M)$ & TCA, PPM, AAM & 16.8 & 18.2 & 22.6 & 31.8 & 1.49 & $<0.01$ & 0.01 \\
\hline Succinate & TCA, BSM, BM & 112.7 & 125.4 & 158.2 & 117.9 & 10.8 & 0.32 & 0.02 \\
\hline \multicolumn{9}{|l|}{ Phenylpropanoid synthesis } \\
\hline Ferulate & PPS, BSM & 17.1 & 20.6 & 13.6 & 20.4 & 1.48 & 0.65 & 0.27 \\
\hline
\end{tabular}

${ }^{1}$ Rumen fluid for the analysis was collected shortly before the morning feeding on d 12 and 21 of each experimental period.

${ }^{2} \mathrm{AAM}=\mathrm{AA}$ metabolism; $\mathrm{AM}=$ acetaldehyde metabolism; APM $=$ arginine and proline metabolism; $\mathrm{BOP}=$ biosynthesis of phenylpropanoids; $\mathrm{BM}=$ butanoate metabolism; $\mathrm{CC}=$ Cori cycle; $\mathrm{CM}=$ caffeine metabolism; $\mathrm{FM}$ = phenylalanine metabolism; GaM = galactose metabolism; $\mathrm{GL}$ = glycolysis; GLM = glycerolipid metabolism; GM = glutathione metabolism; GNG = gluconeogenesis; GPL = glycerophospholipid metabolism; $\mathrm{KBM}=$ ketone bodies metabolism; $\mathrm{MM}=$ methane metabolism; $\mathrm{NM}=$ nitrogen metabolism; PDA = protein digestion and absorption; PM = propanoate metabolism; PPM = purine/pyrimidine metabolism; PPP = pentose phosphate pathway; PPS = phenylpropanoid synthesis; $\mathrm{SMB}$ = secondary metabolite biosynthesis; $\mathrm{SSM}=$ starch and sucrose metabolism; TCA = citrate cycle; TM = tyrosine metabolism; VM = valine metabolism.

${ }^{3}$ Cows were fed once daily at $0800 \mathrm{~h}$.

${ }^{4}$ Linear indicates linear effect of dietary treatment; square indicates a quadratic effect of dietary treatment. 
Table 2. Concentration ( $\mu M$, unless otherwise stated) of rumen metabolites in dairy cows fed graded amounts of barley grain as determined by $\mathrm{GC}-\mathrm{MC}(\mathrm{LSM} \pm \mathrm{SEM} ; \mathrm{n}=8)$

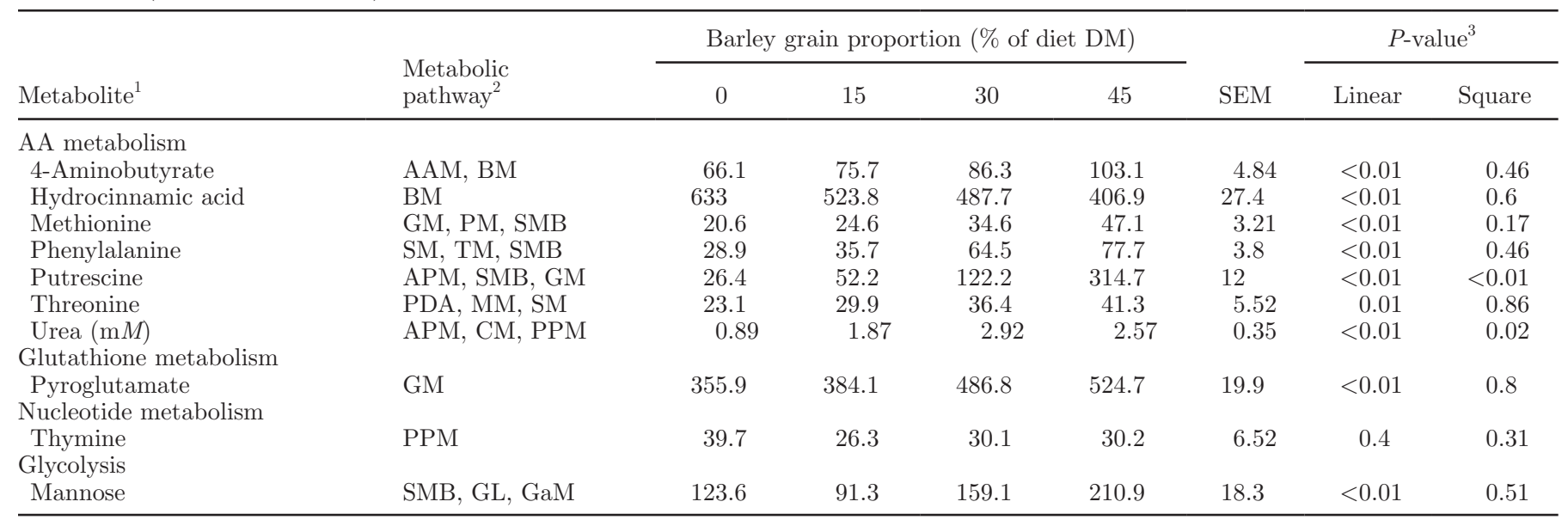

${ }^{1}$ Only metabolites unique to GC-MS are shown. More metabolites were measured but because their concentrations were also measured by nuclear magnetic resonance and direct-injection mass spectrometry and were not found to be statistically different, these data are not given here. Rumen fluid for the analysis was collected shortly before the morning feeding on d 12 and 21 of each experimental period. Cows were fed once daily at $0800 \mathrm{~h}$.

${ }^{2} \mathrm{AAM}=\mathrm{AA}$ metabolism; $\mathrm{APM}=$ arginine and proline metabolism; $\mathrm{BM}=$ butanoate metabolism; $\mathrm{CM}=$ caffeine metabolism; $\mathrm{FM}=$ phenylalanine metabolism; GaM = galactose metabolism; $\mathrm{GL}=$ glycolysis; $\mathrm{GM}=$ glutathione metabolism; $\mathrm{MM}=$ methane metabolism; $\mathrm{PDA}=$ protein digestion and absorption; $\mathrm{PM}=$ propanoate metabolism; $\mathrm{PPM}=$ purine/pyrimidine metabolism; $\mathrm{SM}=$ sphingolipid metabolism; $\mathrm{SMB}$ = secondary metabolite biosynthesis; $\mathrm{TM}=$ tyrosine metabolism.

${ }^{3}$ Linear indicates linear effect of dietary treatment; square indicates a quadratic effect of dietary treatment.

S3A; in the Supplemental Information, available online at http://www.journalofdairyscience.org/). The corresponding PLS-DA loading plot (Figure S3B) indicates that MA, putrescine, hypoxanthine, glucose, and bacterial endotoxin were found to have contributed most to the results of PLS-DA scores plot. The $P$-value for 2,000 permutations was $P<0.001$.

In addition, PLS-DA allowed the identification of the metabolites that were most important for the separation observed in the scores plots, which are shown in Figure 2 (variables with VIP values $>1$ ). Those metabolites that were the most important for the separation of 4 diets (i.e., VIP values $>1$ ) were putrescine, 3-PP, MA, acetate, glucose, hypoxanthine, alanine, ornithine, 1, 3-dihydroxyacetone, histidine, acetoacetate, acylcarnitine 3-hydroxytetradecadienoylcarnitine (C14:2-OH), and phenylalanine. Bacterial endotoxin (LPS) and ruminal $\mathrm{pH}$ were also found to be important (Figure 2). We observed that as the $\mathrm{pH}$ increased, the levels of various organic acids or VFA (acetate, 3-PP, benzoate, and lactate) increased (Figure S5; in the Supplemental Information, available online at http:// www.journalofdairyscience.org/). On the other hand, as the level of LPS increased (and the pH decreased), the quantity of amine-containing compounds (methylamine and putrescine) and bases or base derivatives (uracil and hypoxanthine) increased in ruminal fluid (Figure S6). This indicates that a self-regulating ruminal buffering effect exists arising from the production of acids in high-pH conditions and the production of amines or bases in low-pH conditions.

To visualize the relationship and differences in the concentrations of rumen metabolites among each sample, we used HCA and a heat map representation. The dendrogram in Figure 3 shows the presence of different subclusters corresponding to different numbers of metabolites with various degrees of similarity. The responses of each variable to the 4 diets are indicated with changes in the color intensity on the heat map. Interestingly, the HCA revealed the presence of 1 cluster consisting of a group of phosphatidylcholines (PTC) including PTC ae C36:1, PTC aa C34:2, PTC aa C34:1, PTC aa C36:3, PTC ae C36:2, and PTC ae $\mathrm{C} 40: 3$, where ae $=$ acyl-alkyl and aa $=$ diacyl. An additional subcluster with the HCA that is associated with high-grain diets comprised glucose, putrescine, MA, hypoxanthine, lysine, and alanine, xanthine and glycerol as well as bacterial endotoxin. Another subcluster revealed by HCA was formed by nicotinate along with histidine, proline, caffeine, lactate, phenylacetate, and isoleucine. A distinct feature of the HCA was that rumen metabolites positioned in the top 2 clusters (i.e., from glutamate to 3-hydroxyphenylacetate along with choline to ornithine) of the heat map showed a clear increase with a higher level of grain in the diet. On the other hand, clusters including phosphatidylcholines, in the middle of the heat map, showed a clear decrease with increased grain in the diet. The heat map for the 
Table 3. Concentration ( $\mu M$; unless otherwise stated) of rumen metabolites ${ }^{1}$ in dairy cows fed graded amounts of barley grain as determined by direct flow injection MS/MS (LSM $\pm \mathrm{SEM}, \mathrm{n}=8$ )

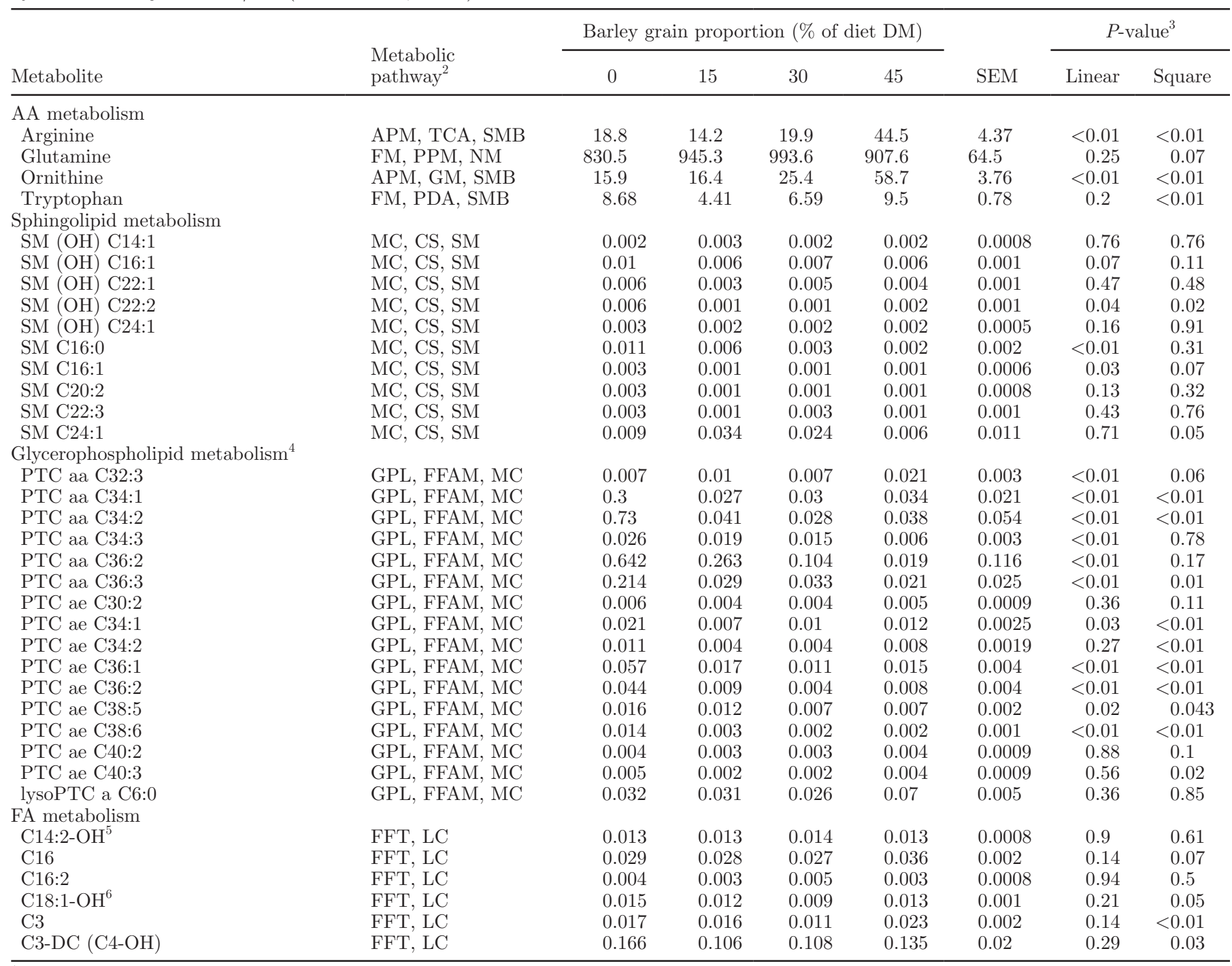

${ }^{1}$ Only metabolites unique to direct flow injection tandem mass spectrometry (MS/MS) are shown. Rumen fluid for the analysis was collected shortly before the morning feeding on d 12 and 21 of each experimental period. Cows were fed once daily at $0800 \mathrm{~h}$.

${ }^{2} \mathrm{APM}=$ arginine and proline metabolism; $\mathrm{CS}=$ cell signaling; FFAM $=$ FFA metabolism; FFT $=$ FA transport; FM $=$ phenylalanine metabolism; GM = glutathione metabolism; GPL = glycerophospholipid metabolism; LC = lipid catabolism; $\mathrm{MC}=$ membrane component; $\mathrm{NM}=$ nitrogen metabolism; PDA = protein digestion and absorption; $\mathrm{PPM}=$ purine/pyrimidine metabolism; $\mathrm{SMB}=$ secondary metabolite biosynthesis; $\mathrm{SM}=$ sphingolipid metabolism; TCA = citrate cycle

${ }^{3}$ Linear indicates linear effect of dietary treatment; square indicates a quadratic effect of dietary treatment.

${ }^{4} \mathrm{PTC}=$ phosphatidylcholine; ae = acyl-alkyl; aa = diacyl; lysoPTC a = lysophosphatidylcholine acyl; and C3-DC = hydroxybutyrylcarnitine. ${ }^{5} 3$-Hydroxytetradecadienoylcarnitine.

${ }^{6} 3$-Hydroxyoleoylcarnitine.

remaining metabolites seemed to be independent of the amount of dietary grain (Figure 3).

\section{Multivariate Data Analysis of Different Measurements Days}

Multivariate data analysis was also used to differentiate between ruminal fluid groups collected at $\mathrm{d} 1$ and 10 of the measurement period (Figure S4; in the Supplemental Information, available online at http:// www.journalofdairyscience.org/). These data revealed interday variations of the rumen metabolite profiles based on the collection day of the ruminal fluid samples (d 1 and 10). The 0 and $30 \%$ barley grain diets did not seem to exhibit any separation of the corresponding clusters, as shown in Figures S4A and S4C, respective- 

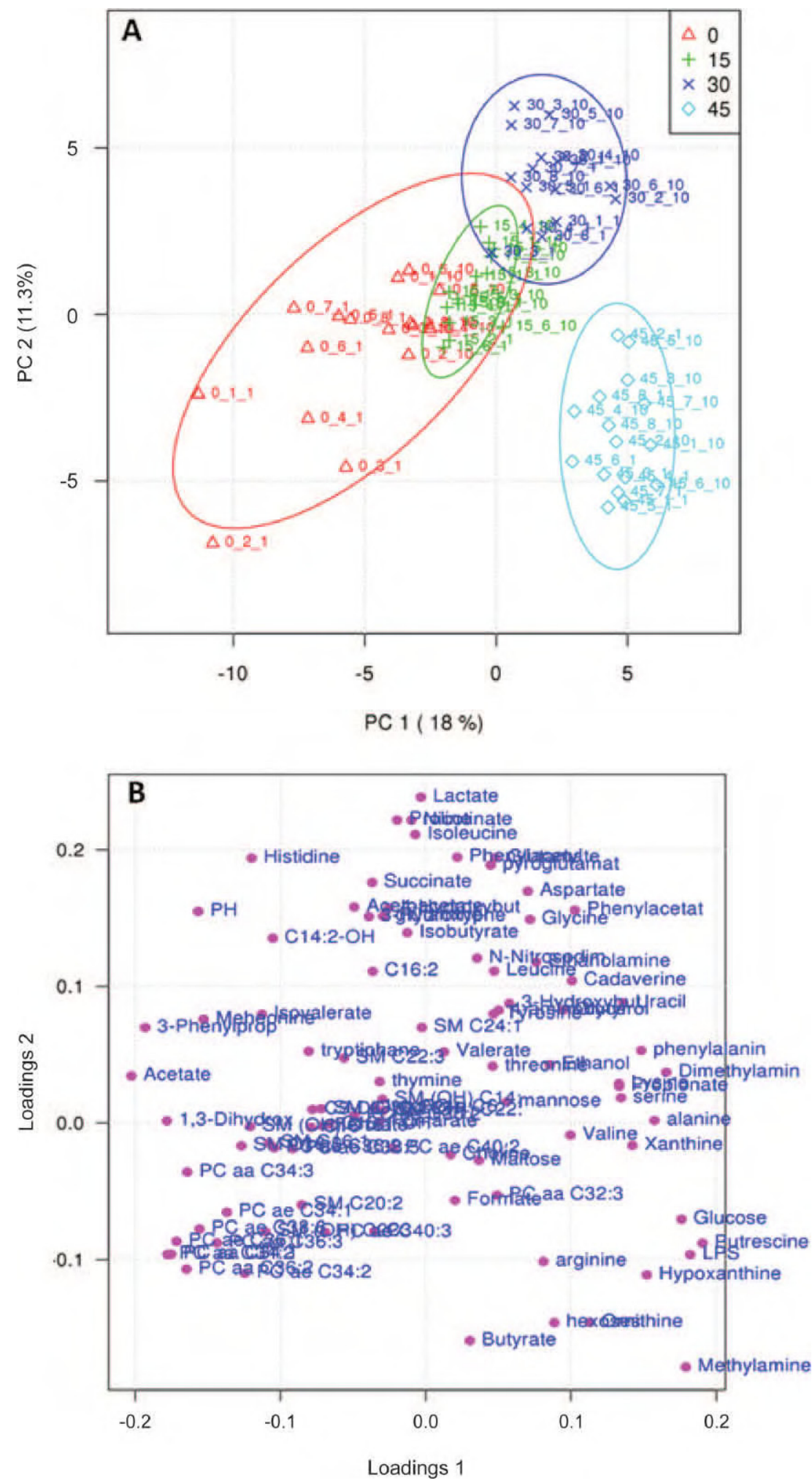

Figure 1. Principal components (PC) analysis (PCA) based on the rumen metabolites profile data. (A) Principal components analysis scores plots discriminating between the rumen fluid of cows fed $0 \%$ (open triangle), $15 \%(+), 30 \%(\mathrm{X})$, and $45 \%$ (open diamond) rolled barely grain in the diet. The first number in the data points represents the diet $(0,15,30$, and 45$)$, the second is the cow number (1-8 cows), and the third indicates the sampling day (1 and 10). (B) Scores plot of the 93 commonly detected metabolites projected into the PCA model. Metabolites are labeled by the names used in Tables 1,2, and 3. Variables with the same distance from 0 with similar positions are positively correlated. Those in the opposite direction are negatively correlated. Color version available in the online PDF. 


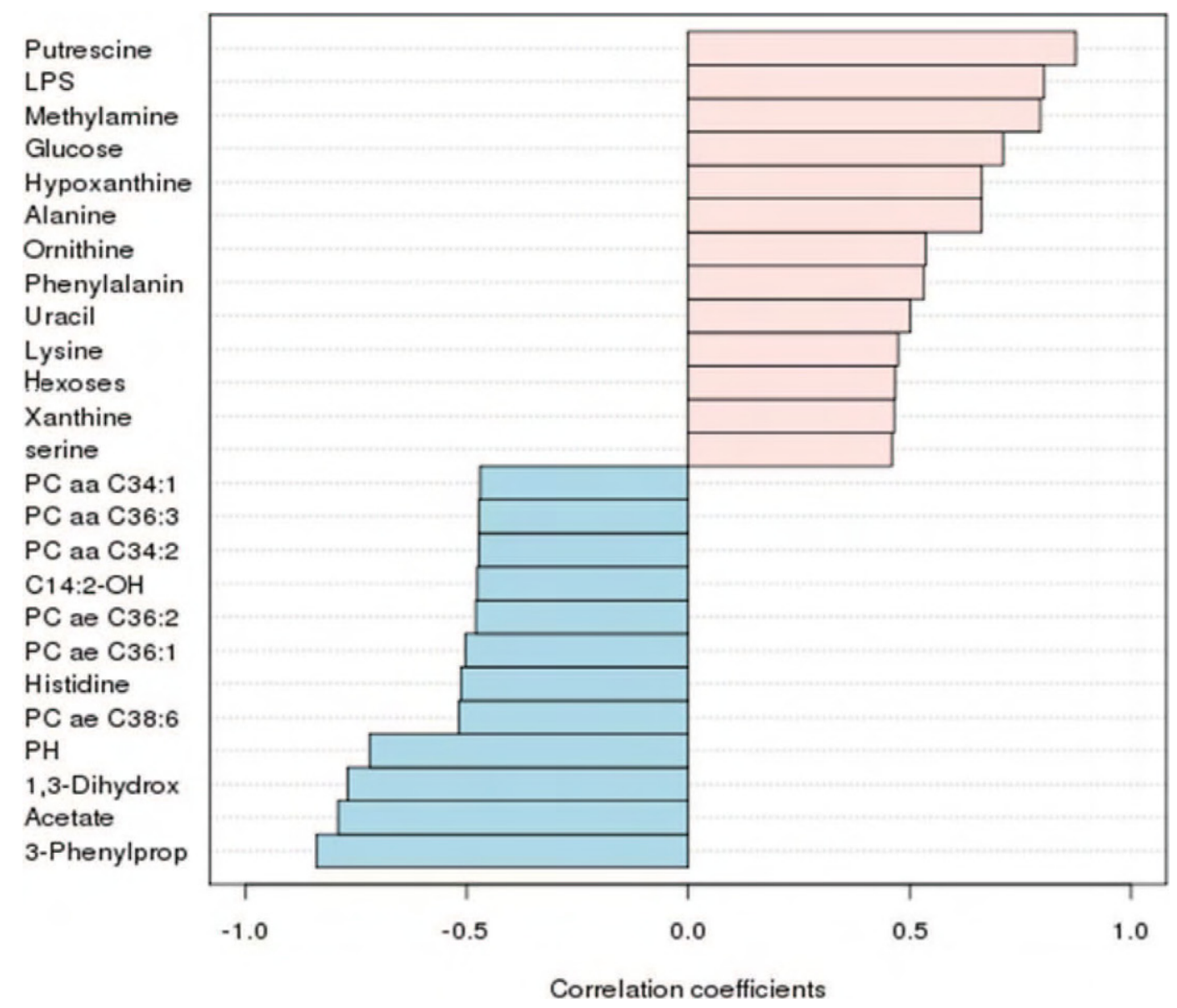

Figure 2. A bar graph showing the top 23 metabolites (along with bacterial endotoxin and ruminal $\mathrm{pH}$ ) correlating with diet $(0,15,30$, and $45 \%$ barley grain). Variables with the same distance from 0 with similar positions are positively correlated. Those in the opposite direction are negatively correlated. $\mathrm{PC}=$ phosphatidylcholine; ae $=$ acyl-alkyl; aa $=$ diacyl; $\mathrm{pH}=$ power of hydrogen (measure of the activity of the hydrogen ion). Color version available in the online PDF.

ly. However, the 15 and $45 \%$ diets revealed 2 distinctive patterns in the corresponding scores plot on Figures S4B and S4D, respectively, which was more evident in the $15 \%$ diet (Figure S4B). The PLS-DA allows for the identification of the metabolites that were important for the separation of these 2 groups at $\mathrm{d} 1$ and 10 .

\section{DISCUSSION}

Previously, we reported that feeding graded amounts of barley grain to dairy cows was associated with major changes in the concentrations of various rumen fluid metabolites (Ametaj et al., 2010b). In that study we used ${ }^{1} \mathrm{H}$ NMR to identify and quantify 46 different metabolites in the rumen fluids of dairy cows. Here, we used 2 more metabolomics platforms (GC-MS and DFI-MS/MS) along with ${ }^{1} \mathrm{H}$ NMR to analyze rumen fluid samples. By using a quantitative, multiplatform approach, the number of measured rumen metabolites was increased to 93 . To our best knowledge, this study is the first comprehensive report that used multiple quantitative metabolomic approaches coupled with multivariate analyses to identify a large number of metabolites and their patterns in the rumen fluid of dairy cows fed graded amounts of grain in their diet. In our discussion, we will focus on metabolite differences between the 4 different grain diets and their potential contribution to the etiopathology of metabolic disorders in dairy cattle due to the feeding of grain-rich diets.

Results from this study confirmed our previous findings that feeding cows 4 different diets $(0,15,30$, and $45 \%$ rolled barley grain) is associated with major shifts in the profile of common as well as uncommon rumen metabolites including SCFA, amino acids, ethanol, endotoxin, and MA (Ametaj et al., 2010b). In addition, the present study revealed major changes in other metabolites that are not typically seen in rumen fluid. These new data both complement and further advance 


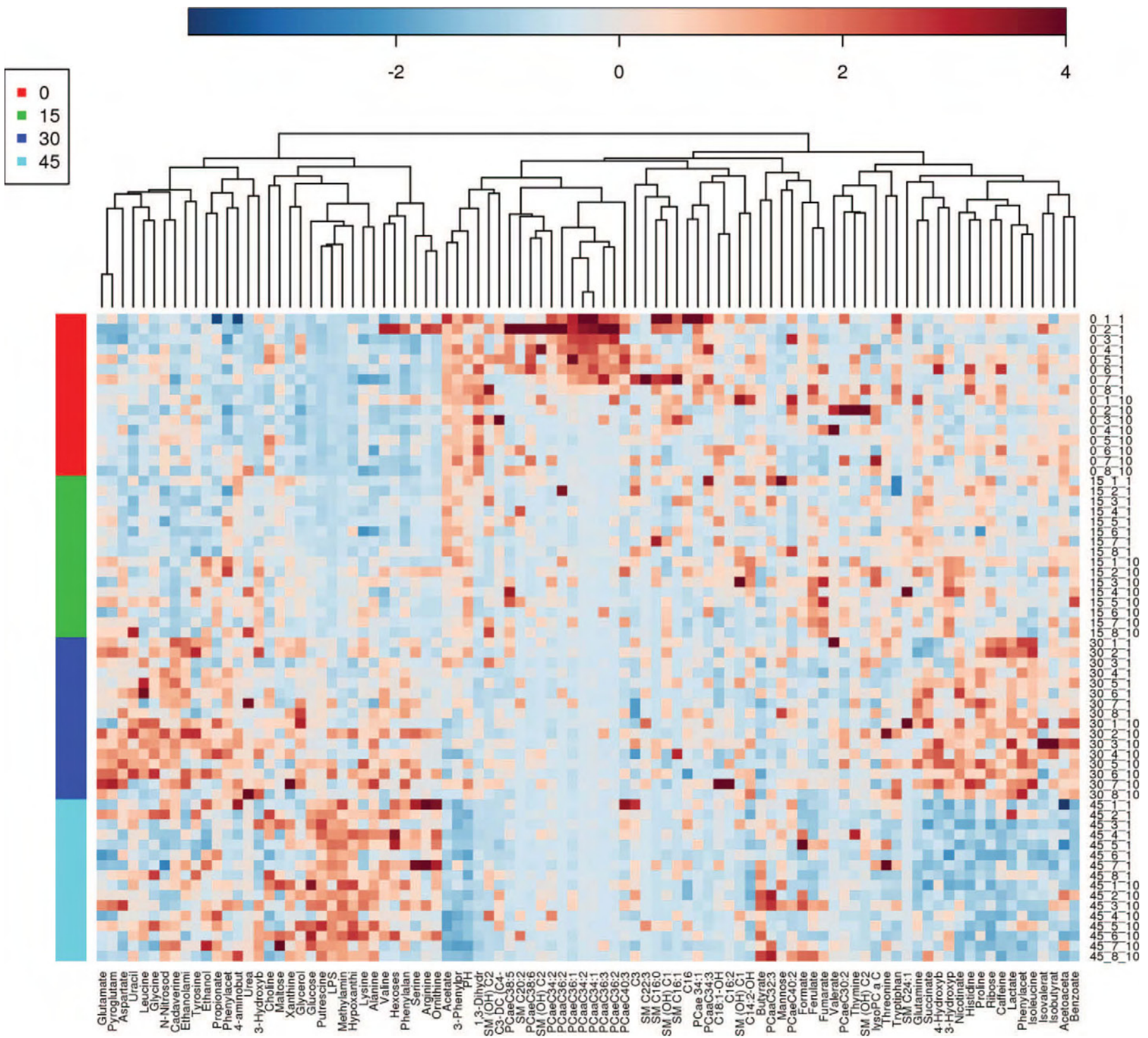

Figure 3. Hierarchical clustering analysis for different rumen fluid metabolites measured before morning feeding in dairy cows fed with 4 different diets $(0,15,30$, and $45 \%$ barley grain inclusion in DM). The expression patterns of each parameter (shown in each row) were categorized by an average linkage hierarchical clustering program (Seo, 2005). Cells are colored based on the signal intensity measured in rumen. Dark brown represents high rumen levels and blue shows low signal intensity and gray cells showing the intermediate level (see color scale above the heat map). The tree clusters and their shorter Euclidean distance indicate higher similarities. Similarity between 2 metabolites is represented by branch height; therefore, the lower a node is vertically, the more similar its subtree. Minimum similarity is $68 \%$. SM $=$ sphingomyelin; PC $=$ phosphatidylcholine; ae = acyl-alkyl; aa = diacyl; and $\mathrm{pH}=$ power of hydrogen; C3-DC = hydroxybutyrylcarnitine.

our knowledge regarding the effects of grain-rich diets on rumen metabolism, especially with regard to rumen and host health.

For example, in this study, a multifold increase in the concentrations of many other biogenic amines besides MA such as putrescine, cadaverine, and dimethylamine was observed. Also, feeding increasing amounts of grain augmented the concentrations of ethanol, N-nitrosodimethylamine, and ethanolamine in the ruminal fluid of dairy cows. Interestingly, these data showed that rumen concentrations of the biogenic amines were greater on d 10 (end of the experiment) as compared with d 1 of the measurement period. The source of biogenic amines in the rumen of cattle is related to diet and 
most importantly to the ruminal $\mathrm{pH}$ and rumen microbiota (Phuntsok et al., 1998). Rumen biogenic amines derive from the decarboxylation of amino acids by the activity of certain types of rumen microbiota (Rice and Koehler, 1976). The most common biogenic amines (i.e., MA, cadaverine, and putrescine) in ruminants are produced from decarboxylation of arginine, lysine, and arginine/ornithine, respectively. Feeding diets rich in grain lowers ruminal $\mathrm{pH}$, and the $\mathrm{pH}$ level in the lumen of the gastrointestinal tract is an important factor that influences the amino acid decarboxylase activity of microbiota. Indeed, amino acid decarboxylase activity has been reported to be greater when the $\mathrm{pH}$ is decreased to an acidotic level (Hill and Mangan, 1964). Data from our study show that cows fed 30 and $45 \%$ barley grain had a rumen $\mathrm{pH}$ below 5.8 for up to 6 to $12 \mathrm{~h}$ postfeeding (Zebeli et al., 2011). Such low pH levels are typically associated with SARA in cows (Stone, 2004). The association between low rumen $\mathrm{pH}$ and a release of large amounts of glucose and amine-containing compounds (MA, putrescine, hypoxanthin, and ornithine) can also be attributed to the effects of low $\mathrm{pH}$ on the diversity of microbiota in the rumen. The sudden availability of easily degradable starch from barley grain, acting as a specific substrate to amylolytic microbiota, lowers rumen $\mathrm{pH}$ and induces a selective and explosive proliferation of certain bacteria species in the rumen. Recent research has demonstrated that switching diets from forage to cereal grains, as done in our study, is associated with increasing counts of starch- and sugardegrading bacteria in the rumen, such as Streptococcus bovis and Lactobacillus spp. (Khafipour et al., 2009). Results of in vitro studies, using equine cecal contents and starch as the carbohydrate source, have shown that streptococci and lactobacilli are the main species having the capacity to decarboxylate certain amino acids and produce biogenic amines (Bailey et al., 2002).

The precise pathophysiological functions of biogenic amines are not yet fully understood. However, due to their structural similarities with endogenous amines, such as the catecholamines, many of the biogenic amines generated in the gastrointestinal tract are hypothesized to have effects on vascular function, if they are translocated into the circulation (Bailey et al., 2003). The latter authors also discussed involvement of gastrointestinal tract-derived biogenic amines in the etiopathology of laminitis in horses fed starch-and fructan-rich diets. Cattle fed large amounts of grain typically are affected by laminitis (Nocek, 1997), but the exact mechanisms behind bovine laminitis in cattle fed grain-rich diets are not well understood. A potential implication of large amounts of biogenic amines produced in the rumen of cows fed large amounts of grain in bovine metabolic disorders such as laminitis needs to be investigated in the future.

Other studies indicate also that biogenic amines are involved in other pathological processes in the host. Intriguingly, studies of putrescine metabolism in animals indicate that the absorbed putrescine is degraded through oxidative deamination, catalyzed by amine oxidases, resulting in the production of aldehyde and hydrogen peroxide (Murooka et al., 1979; Yamashita et al.., 1993). Putrescine serves as a precursor to spermidine and spermine (Matsui and Pegg, 1980), and ruminant animals express plasma amine oxidase, which oxidizes both spermidine and spermine (Blaschko and Bonney, 1962) into aldehyde and hydrogen peroxide (Hölttä et al., 1975). Aldehydes and hydrogen peroxide are known as extremely toxic compounds for a variety of eukaryotic cells and can cause oxidative stress, which may contribute to periparturient disorders and may be associated with metabolic diseases (Ronchi et al., 2000). Besides the toxicity in the systemic circulation, the release of putrescine might also be harmful for the rumen itself. Because the rumen is deficient in catalases, hydrogen peroxide tends to accumulate in the rumen, a result that might be very toxic to ruminal protozoa and rumen epithelial cells (Willard and Kodras, 1967).

One of the most interesting findings from this study is the concentration of ethanolamine and maltose that linearly increased with increasing amounts of barley grain in the diet. To our knowledge, this is the first report demonstrating a direct relationship between feeding large amounts of grain and the release of large amounts of ethanolamine in the rumen fluid of dairy cows. Ethanolamine is a nutrient derived from phosphatidylethanolamine, which is the most abundant phospholipid in membranes of shed enterocytes (Kawai et al., 1974). The exact mechanism(s) regarding how ethanolamine is released in the rumen fluid of cows fed large amounts of grain is not known; however, we hypothesize that the shifts occurring in the microbial activity (Khafipour et al., 2009), and changes in the turnover of epithelial cells (Goodlad, 1981) as well as the cell lysis of ruminal microbiota (Nagaraja et al., 1978) might also be a source of ethanolamine in the rumen in response to grain-rich feeding. Intriguingly, new research has indicated that ethanolamine can be used by pathogenic gram-negative bacteria (enterohemorrhagic E. coli strain O157:H7) as a nitrogen source, conferring a growth advantage over other commensal microbiota. For example, the studies by Bertin et al. (2011) and Thiennimitr et al. (2011) showed that a burst of 2 major pathogenic bacteria such as Salmonella enterica and enterohemorrhagic E. coli was promoted by the presence of ethanolamine in the lumen. So, the 
ability of enterohemorrhagic E. coli strain O157:H7 to use ethanolamine as a nitrogen source provides it with the nutritional and competitive advantage to survive during nutritional stress that leads to human illnesses such as diarrhea, hemolytic-uremic syndrome, and hemorrhagic events (Law, 2000). These diseases are often associated with direct contact through the dairy farm environment, fecal contamination of meat at slaughter, and vegetables that have been fertilized with cattle manure (O'Brien et al., 2001; Yatsuyanagi et al., 2002). These data indicate that the release of such metabolites during periods of nutritional stress might be critical for multiplication of certain pathogenic bacteria with relevance for both animal health and food safety. The fate of ethanolamine is its degradation by bacteria to ethanol and acetate (Bertin et al., 2011). As might be expected, the concentrations of ethanol and acetate were also increased in cows fed large amounts of grain as seen in the current study.

Barley grains are the main source of starch in ruminant diets. Starch is normally hydrolyzed to maltose, a disaccharide (glucose- $\alpha$-glucoside) by enzymatic activity. Catabolism of the disaccharide maltose provides a competitive advantage in vivo to pathogenic $E$. coli O157:H7 during the initiation stage of colonization in intestine that used maltose for its growth. (Jones et al., 2008). A specific maltose-binding protein is located in the periplasmic space of the cell wall of E. coli. The 2 -fold increase in the concentration of ethanolamine and maltose in the rumen of cows fed $45 \%$ barley grain in diets may explain why cattle fed grain had higher populations of this pathogenic E. coli than in cattle fed only hay (Hovde et al., 1999). This may also explain the observation that when these cattle were abruptly switched from a high-grain diet to an all-forage diet, the total populations of $E$. coli decreased 1,000-fold within $5 \mathrm{~d}$. These questions had never been conclusively answered to date.

Another interesting observation arising from this study was the increased rumen concentrations of urea, hypoxanthine, xanthine, uracil, ornithine, LPS, and alanine. These metabolites were associated with feeding of high-grain diets to cows and we found that their concentrations were elevated with increasing proportions of barley grain. All 6 metabolites are degradation products of rumen bacteria as discussed previously (Ametaj et al., 2010b). McAllan and Smith (1973) demonstrated that bacterial nucleic acids (i.e., RNA or DNA) incubated with rumen fluid rapidly degrade into xanthine, hypoxanthine, and uracil. It is also known that the death of both gram-negative and gram-positive bacteria is associated with the release of LPS and alanine (Trent et al., 2006). It is well established that the decrease in the rumen $\mathrm{pH}$ in dairy cows fed high-grain diets is associated with major changes in microbiota composition, as many microbial species are not able to survive the stress of the low pH (Slyter, 1976).

Still another interesting observation from our study that is tied to the low $\mathrm{pH}$ conditions in the rumen is that the rumen fluid concentration of urea rose with increasing levels of grain in the diet. In fact, the concentration of urea peaked at $3 \mathrm{~m} M$ when cows were fed a $45 \%$ barley grain diet. This is consistent with data collected by Broberg (1958) who observed high concentrations of urea in the ruminal fluid of sheep fed grain diets. The presence of urea can be quite problematic, due to its rapid hydrolysis to $\mathrm{NH}_{3}$ by microbial enzymes in the rumen (Golombeski et al., 2006; Highstreet et al., 2010). This is because $\mathrm{NH}_{3}$ can be absorbed through the rumen wall into blood and tissues, where it is toxic. Urea toxicity or high levels of $\mathrm{NH}_{3}$ in the blood can lead to dyspnoea, excessive salivation, frothing, ataxia, weakness, abdominal pain, violent struggling, and bellowing (Blood and Henderson, 1963). Because the concentrations of $\mathrm{CP}$ were similar among the 4 diets of the current study, higher urea concentration in the rumen fluid of dairy cows fed large amounts of grain can be explained either by a higher rate of bacterial degradation or by a more rapid conversion of ammonia to urea as a result of the lower rumen $\mathrm{pH}$ (Aschenbach et al., 2011).

Ornithine also has been shown to be produced by ornithine carbamoyltransferase in the catabolic pathway of arginine in many microorganisms (Crow and Thomas, 1982), including rumen bacteria (Van Den Hende et al., 1964) and protozoa (Onodera et al., 1983). For example, ciliates are known to release ornithine into the ruminal fluid (Onodera and Kandatsu, 1968). Altogether, the increased concentrations of hypoxanthine, xanthine, uracil, alanine, ornithine, ethanolamine, and endotoxin in the rumen fluid of cows on a high-grain diets appears to be a result of the induction of widespread bacterial cell lysis and a fundamental change in the rumen microflora.

We also found high concentrations of glucose in the rumen of dairy cows fed the greatest amounts of barley grain (i.e., 30 and 45\%). Normally, glucose levels are very low in ruminal fluids but higher levels can be found in cattle fed diets high in starch or other rapidly fermentable carbohydrates (McSweeney and Mackie, 1997). Furthermore, the presence of glucose in the rumen fluid of cows fed large amounts of grain might explain why those cows had greater concentrations of lactate in the rumen. Lactate is produced by lactateproducing bacteria such as Streptococcus bovis and Lactobacillus spp., which are more tolerant to the acidic (low pH) conditions. Consequently, these microbes gradually replace other normal rumen microorganisms 
and tend to become the major components of the rumen microbial population by using glucose as their main substrate (Dawson et al., 1997). In our study, besides lactate and propionate, the concentration of butyrate was also increased in the rumen of cows fed large amounts of grain. Butyrate is also produced by the catabolism of lactate in the rumen fluid of cattle fed grain-rich diets in response to a decrease in ruminal $\mathrm{pH}$. In contrast to lactic acidosis, which is characterized by low $\mathrm{pH}$ and increased lactate levels only, SARA is an intermediate state where microbial fermentation is instable and oriented to the production of butyrate, propionate, or both at the expense of acetate (Lettat et al., 2010), as observed in this study. The changes in the SCFA profile in favor of lactate, propionate, and butyrate in response to feeding large amounts of grain arise from changes in the propionate and valerate anabolic pathways of rumen bacteria (Bugaut, 1987). This also arises from shifts in the populations of bacteria and protozoa. For example, Brossard et al. (2004) suggested that the increase in ruminal protozoa concentration could play a central role in butyric SARA by promoting butyrate production from lactate. Greater rumen concentrations of lactate, propionate, and butyrate in response to feeding large amounts of grain found in this study were expected and also agree with previously reported studies (Stone, 2004; Iqbal et al., 2009; Ametaj et al., 2010b).

In addition to substantial changes in the organic acid and $\mathrm{pH}$ profile of rumen fluid from cows on grain-rich diets, we also found a 2 -fold increase in the rumen concentration of ethanol in cows fed 30 and $45 \%$ barley grain diets. This increase in ethanol is due to the high abundance of fermentable carbohydrates in grain that first give rise to glucose, which later converts to ethanol (Orskov and Oltjen, 1967). Another route to the generation of ethanol in the rumen is also from the degradation of ethanolamine (Bertin et al., 2011). Because ethanolamine also was increased in this study with increasing amounts of grain, it is reasonable to suggest that ethanolamine during SARA is also a source of ethanol in the rumen. This is in agreement with previously reported data on ruminants that indicate that overfeeding them with grain is associated with ethanol concentrations in rumen as high as $8 \mathrm{~m} M$ (Allison et al. 1964). Kristensen et al. (2007) also reported rumen ethanol concentrations above $2 \mathrm{~m} M$ in dairy cows fed a TMR containing $54 \%$ corn silage and $13.5 \%$ rolled barley grain. Exposure to high concentrations of ethanol in the lumen of the gastrointestinal tract has been reported to have significant consequences for the host. For example, alcohol exposure can promote the growth of gram-negative bacteria in the intestine, which may result in accumulation of endotoxins (Purohit et al.,
2008). In addition, alcohol metabolism by gramnegative bacteria and intestinal epithelial cells can result in the accumulation of acetaldehyde, which in turn can increase intestinal permeability to endotoxins. Further research is warranted to elucidate the role of ethanol accumulation in the rumen on the permeability of the rumen epithelia to rumen LPS and other toxic compounds that build up in the rumen during SARA. These compounds might have major consequences to the host health.

An interesting metabolite that was revealed by our HCA is nicotinate or niacin. It should be pointed out that nicotinate did not increase with higher proportions of barley grains in the diet (i.e., 45\%). Pike and Brown (1975) found that nicotinate is widely distributed in feeds as niacin in cereals has limited availability for some animal species. Furthermore, the availability of niacin for both host and rumen microbes may be limited when cattle are fed low-roughage diets. Many bacterial species, capable of synthesizing nicotinate, have been identified in rumen contents (e.g., Bacterium ruminicola spp., Bacteroides succinogenes, Ruminococcus flavefaciens, Lachnospira multiparus, Streptococcus bovis, and Butyrivibrio; Menke, 1973). Dietary nicotinate tends to be converted to dinucleotide coenzymes NAD and NADP, which serve as co-substrates in many energy-yielding oxidation-reduction processes (Hankes, 1984). In particular, Hino and Russell (1985) found that NAD favors the deamination of amino acids. Oxidation of NADH to NAD had a marked effect on the deamination of branched-chain amino acids, whereas neutral and charged amino acids tended to be unaffected. So our data suggest that the presence of nicotinate in the rumen favors the deamination of amino acids, which may partly explain the elevated ruminal levels of biogenic amines noted earlier.

Whereas many rumen metabolites increased with increasing levels of grain in the diet, a few compounds were found to decrease. In particular, our data indicated that the concentration of 3-PP linearly decreased with increasing proportions of grain in the diet. In contrast, the opposite effect was observed with regard to ruminal fluid concentrations of phenylacetate. This result confirms previous findings by our team where feeding cows large amounts of grain was associated with lowered levels of 3-PP (Ametaj et al., 2010b). Research conducted in forage-fed sheep has shown that the latter 2 compounds are important aromatic acids in ruminal fluid, with 3-PP and phenylacetate accounting for about 50.8 and $13.5 \%$ of total aromatic acids in the rumen fluid, respectively (Pagella, 1998). Our results demonstrated that the ratio between these aromatic compounds could change with increasing amounts of grain in the diet. Phenylacetate and 3-PP in the rumen are generated 
by the hydrogenation activity of ruminal microbes on plant phenolic compounds such as $p$-coumaric, ferulic, and caffeic acids, followed by a dehydroxylation process (Chesson et al., 1999). An alternative origin of 3-PP and phenylacetate is also possible via the deamination of aromatic amino acids such as tyrosine and phenylalanine (Martin, 1982; Burlingame and Chapman, 1983), but to our knowledge no studies have linked the concentration changes of 3-PP and phenylacetate to the overall health of the dairy cattle.

Another interesting cluster of metabolites, as revealed by HCA and associated with high-grain diets was a group of 6 PTC compounds (i.e., PTC aa C34:1, PTC aa C34:2, PTC ae C36:2, PTC ae C36:1, and PTC ae C38:6), where ae = acyl-alkyl and aa = diacyl. Concentrations of these compounds decreased with increasing amounts of barley grain in the diet. The very low concentrations of PTC in the ruminal fluid could be related to the time of sampling, which was performed before the morning feeding. Phosphatidylcholine is a major phospholipid in ruminants and it is critical for lipid absorption and transport, cell membrane structure, cell signaling, and synthesis of lipoproteins (Zeisel and Holmes-McNary, 2001). Phosphatidylcholine is needed for the synthesis of very low-density lipoproteins, which are responsible for the export of triacylglycerol from hepatocytes. Low levels of choline are often blamed for the accumulation of triglycerides in the liver and development of fatty liver disease in dairy cattle (Piepenbrink and Overton 2003). The main dietary source of choline in ruminant diets is PTC from plant membrane material (Neill et al., 1978), microbial choline, and choline supplements.

Choline that reaches the small intestine of cows is in the form of PTC, the main phospholipid in the rumen protozoa (Broad and Dawson, 1976). Phosphatidylcholine is present in rumen protozoa as a major component of their cell membranes. Phosphatidylcholines comprise about $3 \%$ of the total phospholipids, whereas protozoal biomass accounts for 20 to $50 \%$ of the total microbial biomass in the rumen (Coleman, 1979). It is assumed that most of the PTC metabolites in the rumen fluid come from protozoa. The density of protozoa in the rumen is correlated with the diet of the animals. It has been reported that grain-rich diets are associated with lowered counts of protozoa in the rumen (Jouany et al., 1989). Results of the study by Goad et al. (1998) and Khafipour et al. (2009) showed decreases in the number of protozoa in cows during SARA. Thus, it is suggested that concentration of rumen PTC compounds could be used as biomarkers indicating a decrease of rumen protozoa counts when cows are fed diets with high grain content.
Our study also revealed a temporal variation in the rumen concentration of metabolites occurring between d 1 and 10 of the measurement period, particularly for the diets with the highest levels of grain. Multivariate analysis demonstrated that ruminal fluid collected on $\mathrm{d}$ 1 and 10 from cows fed 15 and $45 \%$ barley grain could be easily separated on the basis of their metabolite concentrations. The 10 most important metabolites that contribute to this interday separation were aspartate, methylamine, putrescine, LPS, alanine, glucose, ferulate, and formate. Their concentrations were increased at d 10 compared with $\mathrm{d} 1$, whereas the concentration of 3-PP and the $\mathrm{pH}$ were found to be lower at $\mathrm{d} 10$. These data suggest that high-grain diets $(>30 \%)$ require longer periods of physiological adaptation than low-grain diets. In a previous study, in which yearling steers were fed $70,75,80$, and $85 \%$ concentrate diets based on steam-flaked corn for $5 \mathrm{~d}$ each followed by a $90 \%$ concentrate, the ruminal fluid metabolic profiles showed a clear separation at d 1 and 10 (Choat et al., 2002). Bevans et al. (2005) transitioned heifers from a $40 \%$ concentrate diet to a $90 \%$ concentrate diet by feeding a diet containing $65 \%$ concentrate for $3 \mathrm{~d}$ only. The mean ruminal $\mathrm{pH}$ did not differ during the first day but $\mathrm{pH}$ became more acidic on $\mathrm{d} 2$ and 3 after introduction of the $65 \%$ concentrate diet. The results from our current study indicate that $21 \mathrm{~d}$ of feed intake from $45 \%$ barley grain was not enough for full diet adaptation and might lead to rumen acidosis in cows.

\section{CONCLUSIONS}

As high productivity is expected from dairy cows, the use of readily fermentable diets becomes necessary to meet their very high energy and protein requirements. However, this comes at a cost that leads to significant metabolic imbalances in the rumen and surrounding tissues, which likely contribute to the observed increase in periparturient diseases. Our data show, unequivocally, that increasing amounts of cereal grain in the diet of lactating cows leads to marked increases in the rumen fluid concentrations of putrescine, methylamines, and other biogenic amines. It also leads to increased ruminal acidity and increased levels and endotoxin. High grain consumption also leads to high levels of glucose, alanine, maltose, ornithine, propionate, uracil, valerate, xanthine, and phenylacetate as well as to potentially increased concentrations of $N$-nitrosodimethylamine (NDMA), urea, ethanolamine, maltose, and dimethylamine (DMA). These changes indicate that significant alterations to the rumen microflora are taking place and that these are reflected by widespread cell death (manifested by altered levels of purine metabolites and 
certain amino acids), the release of harmful or proinflammatory compounds (endotoxin, urea/ $\mathrm{NH}_{3}$, ethanol, and biogenic amines) into the rumen, and the production of VFA (leading to the acidification of the rumen). The acidification of the rumen also increases intestinal permeability to these compounds. Ruminal acidification due to high grain consumption also leads to further cell death, further microfloral changes, and the release of even more harmful compounds, thereby setting up a vicious cycle not unlike that seen in human gastrointestinal diseases or chronic inflammatory conditions such as ulcerative colitis and Crohn's disease. In addition to these metabolic changes, we have also highlighted how some rumen-derived metabolites (particularly those associated with high-grain diets) have been previously associated with elevated risks or higher incidences of certain periparturient diseases such as SARA, acute rumen acidosis, urea toxicity, fatty liver disease, as well as inflammatory diseases such as mastitis, laminitis, milk fever, and other conditions. These data certainly underline the importance of gaining a better understanding of the biochemical function of rumen as a whole ecosystem. Improvements in our understanding between diet and rumen health, as well as improved methods for monitoring these changes, will enable us to maintain the fine balance between high milk productivity and good herd health.

\section{ACKNOWLEDGMENTS}

This work is part of a project entitled "Profiling of Dairy Cattle Metabolome" co-led by B. N. Ametaj and D. S. Wishart, which was supported financially by the Alberta Agricultural Research Institute (AARI; Edmonton, AB, Canada), the Alberta Livestock and Meat Agency (ALMA; Edmonton, AB, Canada), and the Natural Sciences and Engineering Research Council of Canada (NSERC; Ottawa, ON, Canada). The technical assistance of D. G. V. Emmanuel, R. P. Pandian, and S. Sivaraman (University of Alberta, Edmonton, AB, Canada) is highly appreciated. We also are grateful to the technical staff of Dairy Research and Technology Centre at the University of Alberta (Edmonton, AB, Canada) for their help and care to the cows used in this study.

\section{REFERENCES}

Allison, M. J., R. W. Dougherty, J. A. Bucklin, and E. E. Snyder. 1964. Ethanol accumulation in the rumen after overfeeding with readily fermentable carbohydrate. Science 144:54-55.

Ametaj, B. N., Q. Zebeli, and S. Iqbal. 2010a. Nutrition, microbiota, and endotoxin-related diseases in dairy cows. R. Bras. Zootec. 39:433-444.
Ametaj, B. N., Q. Zebeli, F. Saleem, N. Psychogios, M. J. Lewis, S. M. Dunn, J. Xia, and D. S. Wishart. 2010b. Metabolomics reveals unhealthy alterations in rumen metabolism with increased proportion of cereal grain in the diet of dairy cows. Metabolomics 6:583-594.

Aschenbach, J. R., G. B. Penner, F. Stumpff, and G. Gäbel. 2011. Ruminant Nutrition Symposium: Role of fermentation acid absorption in the regulation of ruminal pH. J. Anim. Sci. 89:1092-1107.

Bailey, S. R., C. M. Marr, and J. Elliott. 2003. Identification and quantification of amines in the equine caecum. Res. Vet. Sci. 74:113-118.

Bailey, S. R., A. Rycroft, and J. Elliott. 2002. Production of amines in equine cecal contents in an in vitro model of carbohydrate overload. J. Anim. Sci. 80:2656-2662.

Bertin, Y., J. P. Girardeau, F. Chaucheyras-Durand, B. Lyan, E. Pujos-Guillot, J. Harel, and C. Martin. 2011. Enterohaemorrhagic Escherichia coli gains a competitive advantage by using ethanolamine as a nitrogen source in the bovine intestinal content. Environ. Microbiol. 13:365-377.

Bevans, D. W., K. A. Beauchemin, K. S. Schwartzkopf-Genswein, J. J. McKinnon, and T. A. McAllister. 2005. Effect of rapid or gradual grain adaptation on subacute acidosis and feed intake by feedlot cattle. J. Anim. Sci. 83:1116-1132.

Blaschko, H., and R. Bonney. 1962. Spermine oxidase and benzylamine oxidase. Distribution, development and substrate specificity. Proc. R. Soc. Lond. 156:268-279.

Blood, D. C., and J. A. Henderson. 1963. Veterinary Medicine. 2nd ed. Page 107. Baillière, Tyndall \& Cassell, London, UK.

Broad, T. E., and R. M. C. Dawson. 1976. Role of choline in the nutrition of the rumen protozoon Entodinium caudatum. J. Gen. Microbiol. 92:391-397.

Broberg, G. 1958. Studies on the effect of over-feeding in sheep. Pages 365-369 in Proc. 8th Nordic Vet. Cong. Pergamon Press, Oxford, UK.

Brossard, L., C. Martin, F. Chaucheyras-Durand, and B. MichaletDoreau. 2004. Protozoa involved in butyric rather than lactic fermentative pattern during latent acidosis in sheep. Reprod. Nutr. Dev. 44:195-206.

Bugaut, M. 1987. Occurrence, absorption and metabolism of short chain fatty acids in the digestive tract of mammals. Comp. Biochem. Physiol. B 86:439-472.

Burlingame, R., and P. J. Chapman. 1983. Catabolism of phenylpropionic acid and its 3-hydroxy derivative by Escherichia coli. J. Bacteriol. 155:113-121.

CCAC (Canadian Council on Animal Care). 1993. Guide to the Care and Use of Experimental Animals. 2nd ed. Vol. 1. CCAC, Ottawa, ON, Canada.

Chesson, A., G. J. Provan, W. R. Russell, L. Scobbie, A. J. Richardson, and C. Stewart. 1999. Hydroxycinnamic acids in the digestive tract of livestock and humans. J. Sci. Food Agric. 79:373-378.

Choat, W. T., C. R. Krehbiel, M. S. Brown, G. C. Duff, D. A. Walker, and D. R. Gill. 2002. Effects of restricted versus conventional dietary adaptation on feedlot performance, carcass characteristics, site and extent of digestion, digesta kinetics, and ruminal metabolism. J. Anim. Sci. 80:2726-2739.

Coleman, G. S. 1979. The role of rumen protozoa in the metabolism of ruminants given tropical feeds. Trop. Anim. Prod. 4:199-213.

Crow, V. L., and T. D. Thomas. 1982. Arginine metabolism in lactic streptococci. J. Bacteriol. 150:1024-1032.

Dawson, K. A., M. A. Rasmussen, and M. J. Allison. 1997. Digestive disorders and nutritional toxicity. Pages $631-660$ in The Rumen Microbial Ecosystem, 2nd ed. P. N. Hobson, and C. S. Stewart, ed. Chapman \& Hall, London, UK.

Dieterle, F., A. Ross, G. Schlotterbeck, and H. Senn. 2006. Probabilistic quotient normalization as robust method to account for dilution of complex biological mixtures. Application in ${ }^{1} \mathrm{H}$ NMR metabonomics. Anal. Chem. 78:4281-4290.

Emmanuel, D. G. V., S. M. Dunn, and B. N. Ametaj. 2008. Feeding high proportions of barley grain stimulates an inflammatory response in dairy cows. J. Dairy Sci. 91:606-614. 
Fernando, S. C., H. T. Purvis, F. Z. Najar, L. O. Sukharnikov, C. R. Krehbiel, T. G. Nagaraja, B. A. Roe, and U. DeSilva. 2010. Rumen microbial population dynamics during adaptation to a highgrain diet. Appl. Environ. Microbiol. 76:7482-7490.

Goad, D. W., C. L. Goad, and T. G. Nagaraja. 1998. Ruminal microbial and fermentative changes associated with experimentally induced subacute acidosis in steers. J. Anim. Sci. 76:234-241.

Golombeski, G. L., K. F. Kalscheur, A. R. Hippen, and D. J. Schingoethe. 2006. Slow-release urea and highly fermentable sugars in diets fed to lactating dairy cows. J. Dairy Sci. 89:4395-4403.

Goodlad, R. A. 1981. Some effects of diet on the mitotic index and the cell cycle of the ruminal epithelium of sheep. Q. J. Exp. Physiol. 66:487-499.

Hankes, L. V. 1984. Nicotinic acid and nicotinamide. Pages 329-377 in Handbook of Vitamins, Nutritional, Biochemical and Clinical Aspects. L. J. Machlin, ed. Marcel Dekker Inc., New York, NY.

Highstreet, A., P. H. Robinson, J. Robison, and J. G. Garrett. 2010. Response of Holstein cows to replacing urea with a slowly rumen released urea in a diet high in soluble crude protein. Livest. Sci. 129:179-185.

Hill, K. J., and J. Mangan. 1964. The formation and distribution of methylamine in the ruminant digestive tract. Biochem. J. 93:3945.

Hino, T., and J. B. Russell. 1985. The effect of reducing equivalent disposal and NADH/NAD on the deamination of amino acids by intact and cell-free extracts of rumen microorganisms. Appl. Environ. Microbiol. 50:1368-1374.

Hölttä, E., P. Pulkkinen, K. Elfving, and J. Jänne. 1975. Oxidation of polyamines by diamine oxidase from human seminal plasma. Biochem. J. 145:373-378.

Hovde, C. J., P. R. Austin, K. A. Cloud, C. J. Williams, and C. W Hunt. 1999. Effect of cattle diet on Escherichia coli O157:H7 acid resistance. Appl. Environ. Microbiol. 65:3233-3235

Iqbal, S., Q. Zebeli, A. Mazzolari, G. Bertoni, S. M. Dunn, W. Z. Yang, and B. N. Ametaj. 2009. Feeding barley grain steeped in lactic acid modulates rumen fermentation patterns and increases milk fat content in dairy cows. J. Dairy Sci. 92:6023-6032.

Jiye, A., J. Trygg, J. Gullberg, A. I. Johansson, P. Jonsson, H. Antti, S. L. Marklund, and T. Moritz. 2005. Extraction and GC/MS analysis of the human blood plasma metabolome. J. Anal. Chem. 77:8086-8094.

Jones, S. A., M. Jorgensen, F. Z. Chowdhury, R. Rodgers, J. Hartline, M. P. Leatham, C. Struve, K. A. Krogfelt, P. S. Cohen, and T. Conway. 2008. Glycogen and maltose utilization by Escherichia coli O157:H7 in the mouse intestine. Infect. Immun. 76:2531-2540.

Jouany, J. P., D. I. Demeyer, and J. Grain. 1988. Effect of defaunating the rumen. Anim. Feed Sci. Technol. 21:229-265.

Kawai, K., M. Fujita, and M. Nakao. 1974. Lipid components of two different regions of an intestinal epithelial cell membrane of mouse. Biochim. Biophys. Acta 369:222-233.

Khafipour, E., S. Li, J. C. Plaizier, and D. O. Krause. 2009. Rumen microbiome composition determined using two nutritional models of subacute ruminal acidosis. Appl. Environ. Microbiol. 75:7115-7124.

Kristensen, N. B., A. Storm, B. M. Raun, B. A. Røjen, and D. L. Harmon. 2007. Metabolism of silage alcohols in lactating dairy cows. J. Dairy Sci. 90:1364-1377.

Law, D. 2000. Virulence factors of Escherichia coli O157 and other Shiga toxin-producing E. coli. J. Appl. Microbiol. 88:729-745.

Lettat, A., P. Nozière, M. Silberberg, D. P. Morgavi, C. Berger, and C. Martin. 2010. Experimental feed induction of ruminal lactic, propionic, or butyric acidosis in sheep. J. Anim. Sci. 88:3041-3046.

Martin, A. K. 1982. The origin of urinary aromatic compounds excreted by ruminants 3 . The metabolism of phenolic compounds to simple phenols. Br. J. Nutr. 48:497-507.

Matsui, I., and A. E. Pegg. 1980. Increase in acetylation of spermidine in rat liver extracts brought about by treatment with carbon tetrachloride. Biochem. Biophys. Res. Commun. 92:1009-1015.

McAllan, A. B., and R. H. Smith. 1973. Degradation of nucleic acids in the rumen. Br. J. Nutr. 29:331-345.
McSweeney, C. S., and R. I. Mackie. 1997. Gastrointestinal detoxification and digestive disorders in ruminant animals. Pages 583-634 in Gastrointestinal Microbiology. R. I. Mackie and B. A. White, ed. Chapman and Hall, New York, NY.

Menke, K. H. 1973. Vitamin syntheses in the rumen. Pages 235-255 in Biology and Biochemistry of Microbial Digestion. D. Giesecke and H. K. Henderickx, ed. BLV Verlagsgesellschaft, Munich, Germany. Murooka, Y., N. Doi, and T. Harada. 1979. Distribution of membranebound monoamine oxidase in bacteria. Appl. Environ. Microbiol. 38:565-569.

Nagaraja, T. G., E. E. Bartley, L. R. Fina, and H. D. Anthony. 1978. Relationship of rumen gram-negative bacteria and free endotoxin to lactic acidosis in cattle. J. Anim. Sci. 47:1329-1337.

Neill, A. R., D. W. Grime, and R. M. C. Dawson. 1978. Conversion of choline methyl groups through trimethylamine into methane in the rumen. Biochem. J. 170:529-535.

Nocek, J. E. 1997. Bovine acidosis: Implications on laminitis. J. Dairy Sci. 80:1005-1028.

NRC. 2001. Nutrient Requirements of Dairy Cattle. 7th rev. ed. National Academy of Sciences, Washington, DC.

O'Brien, S. J., G. K. Adak, and C. Gilham. 2001. Contact with farming environment as a major risk factor for Shiga toxin (verocytotoxin)producing Escherichia coli O157 infection in humans. Emerg. Infect. Dis. 7:1049-1051.

Onodera, R., and M. Kandatsu. 1968. Amino acid and protein metabolism of rumen ciliate protozoa. 1. Consumption of amino acids. Jpn. J. Zootech. Sci. 39:206-211.

Onodera, R., Y. Yamaguchi, and S. Morimoto. 1983. Metabolism of arginine, citrulline, ornithine, and proline by starved rumen ciliate protozoa. Agric. Biol. Chem. 47:821-828.

Orskov, E. R., and R. R. Oltjen. 1967. Influence of carbohydrate and nitrogen sources on the rumen volatile fatty acids and ethanol of cattle fed purified diets. J. Nutr. 93:222-228.

Pagella, J. H. 1998. Urinary benzylated compounds as potential markers of forage intake and metabolism of their precursors in ruminants. PhD Diss. Aberdeen University, Aberdeen, UK.

Phuntsok, T., M. A. Froetschel, H. E. Amos, M. Zheng, and Y. W. Huang. 1998. Biogenic amines in silage, apparent post-ruminal passage, and the relationship between biogenic amines and digestive function and intake by steers. J. Dairy Sci. 81:2193-2203.

Piepenbrink, M. S., and T. R. Overton. 2003. Liver metabolism and production of cows fed increasing amounts of rumen-protected choline during the periparturient period. J. Dairy Sci. 86:1722-1733.

Pike, R. L., and M. L. Brown. 1975. Nutrition: An Integrated Approach. 2nd ed. John Wiley and Sons, New York, NY.

Psychogios, N., D. D. Hau, J. Peng, A. C. Guo, and R. Mandal. 2011. The human serum metabolome. PLoS ONE 6:e16957.

Purohit, V., J. C. Bode, C. Bode, D. A. Brenner, M. A. Choudhry, F. Hamilton, Y. J. Kang, A. Keshavarzian, R. Rao, R. B. Sartor, C. Swanson, and J. R. Turner. 2008. Alcohol, intestinal bacterial growth, intestinal permeability to endotoxin, and medical consequences. Alcohol 42:349-361.

Rice, S. L., and P. E. Koehler. 1976. Tyrosine and histidine decarboxylase activities of Pediococcus cerevisiae and Lactobacillus species and the production of tyramine in fermented sausages. J. Milk Food Technol. 39:166-169.

Ronchi, B., U. Bernabucci, N. Lacetera, and A. Nardone. 2000. Oxidative and metabolic status of high yielding dairy cows in different nutritional conditions during the transition period. Page 125 in Proc. 51st Annual Mtg. EAAP, Vienna, Austria. Wagningen Pers., Wageningen, the Netherlands.

Seo, J. 2005. Information visualization design for multidimensional data: integrating the rank-by-feature framework with hierarchical clustering. PhD Diss. University of Maryland, College Park.

Slyter, L. L. 1976. Influence of acidosis on rumen function. J. Anim. Sci. 43:910-929.

Stone, W. C. 2004. Nutritional approaches to minimize subacute ruminal acidosis and laminitis in dairy cattle. J. Dairy Sci. 87(E. Suppl.):E13-E26.

Szeto, S. S., S. N. Reinke, B. D. Sykes, and B. D. Lemire. 2010. Mutations in the Saccharomyces cerevisiae succinate dehydrogenase 
result in distinct metabolic phenotypes revealed through ${ }^{1} \mathrm{H}$ NMRbased metabolic footprinting. J. Proteome Res. 9:6729-6739.

Thiennimitr, P., S. E. Winter, M. G. Winter, M. N. Xavier, V. Tolstikov, D. L. Huseby, T. R. Sterzenbach, M. Tsolis, J. R. Roth, and A. J. Bäumler. 2011. Intestinal inflammation allows Salmonella to use ethanolamine to compete with the microbiota. Proc. Natl. Acad. Sci. USA 108:17480-17485.

Trent, M. S., C. M. Stead, A. X. Tran, and J. V. Hankins. 2006. Diversity of endotoxin and its impact on pathogenesis. J. Endotoxin Res. 12:205-223.

Van Den Hende, C., W. Oyaert, and J. H. Bouckaert. 1964. The metabolism of arginine, citrulline, ornithine, proline and delta-amino valeric acid and phenylalanine by rumen bacteria. Res. Vet. Sci. $5: 491-496$.

Willard, F. L., and R. Kodras. 1967. Survey of chemical compounds tested in vitro against rumen protozoa for possible control of bloat. Appl. Microbiol. 15:1014-1019.

Xia, J., N. Psychogios, N. Young, and D. S. Wishart. 2009. MetaboAnalyst: A web server for metabolomic data analysis and interpretation. Nucleic Acids Res. 37:W652-W660.
Yamashita, Y., W. H. Bowen, R. A. Burne, and H. K. Kuramitsu. 1993. Role of the Streptococcus mutans gtf genes in caries induction in the specific-pathogen-free rat model. Infect. Immun. 61:38113817.

Yatsuyanagi, J., S. Saito, and I. Ito. 2002. A case of hemolytic-uremic syndrome associated with Shiga toxin 2-producing Escherichia coli O121 infection caused by drinking water contaminated with bovine feces. Jpn. J. Infect. Dis. 55:174-176.

Zebeli, Q., J. Dijkstra, M. Tafaj, H. Steingass, B. N. Ametaj, and W. Drochner. 2008. Modeling the adequacy of dietary fiber in dairy cows based on the responses of ruminal $\mathrm{pH}$ and milk fat production to composition of the diet. J. Dairy Sci. 91:2046-2066.

Zebeli, Q., S. M. Dunn, and B. N. Ametaj. 2011. Perturbation of plasma metabolites correlated with the rise of rumen endotoxin in dairy cows fed diets rich in easily degradable carbohydrates. J. Dairy Sci. 94:2374-2382.

Zeisel, S. H., and M. Holmes-McNary. 2001. Choline. Pages 513-528 in Handbook of Vitamins. 3rd ed. Marcel Dekker Inc., New York, NY. 